Age $<20$ years was associated with NMDA-R-IgG and MOG-IgG1 $(\mathrm{OR}=8.11$ and 7.73 respectively, $\mathrm{p}<0.001)$. Age $>65$ years was associated with GABAB-R-IgG, LGI1-IgG, CASPR2-IgG and ANNA1-IgG (OR=7.33, 14.98, 3.67, 14.53, $\mathrm{p}<0.001)$. Women accounted for $60 \%$ of NMDA-R-IgG (CSF) and $78 \%$ of GAD65-IgG (CSF/serum) cohorts $(\mathrm{OR}=1.32$, $\mathrm{p}=0.002, \mathrm{OR}=2.78, \mathrm{p}<0.001$, respectively). Men accounted for $62 \%$ of the LGI1-IgG cohort $(\mathrm{OR}=1.87, \mathrm{p}<0.001)$. Age and sex interacted for NMDA-R-IgG, particularly in females $<20$ years $(\mathrm{OR}=7.72, \mathrm{p}<0.001)$.

Conclusion The most frequently detected were NMDA-R-IgG, LGI1-IgG, GAD65-IgG and MOG-IgG1. Age and sex associations may suggest paraneoplastic, endocrinological or aging influences on neurological autoimmunity.

\section{PREGNANCY-RELATED RELAPSE IN NATALIZUMAB, FINGOLIMOD AND DIMETHYL FUMARATE-TREATED WOMEN WITH MULTIPLE SCLEROSIS}

1,2Wei Z Yeh, ${ }^{1}$ Putu A Widyastuti, ${ }^{1,2}$ Anneke Van der Walt, ${ }^{1}$ Jim Stankovich, ${ }^{3}$ Eva K Havrdova, ${ }^{3}$ Dana Horakova, ${ }^{3}$ Karolina Vodehnalova, ${ }^{4}$ Serkan Ozakbas, ${ }^{5}$ Sara Eichau, ${ }^{6}$ Pierre Duquette, ${ }^{8,7}$ Tomas Kalincik, ${ }^{9}$ Francesco Patti, ${ }^{10}$ Cavit Boz, ${ }^{11}$ Murat Terzi, ${ }^{12}$ Bassem Yamout, ${ }^{13}$ Jeannette Lechner-Scott, ${ }^{14}$ Patrizia Sola, ${ }^{2}$ Olga Skibina, ${ }^{15}$ Michael Barnett, ${ }^{16}$ Marco Onofrj, ${ }^{17}$ Maria J Sá, ${ }^{18,19}$ Pamela McCombe, ${ }^{20}$ Pierre Grammond, ${ }^{21}$ Radek Ampapa, ${ }^{22}$ Francois Grand'Maison, ${ }^{23}$ Roberto Bergamaschi, ${ }^{24}$ Daniele LA Spitaleri, ${ }^{25}$ Vincent Van Pesch, ${ }^{26}$ Elisabetta Cartechini, ${ }^{27}$ Suzanne Hodgkinson, ${ }^{28}$ Aysun Soysal, ${ }^{29}$ Albert Saiz, ${ }^{1,2}$ Melissa Gresle, ${ }^{3}$ Tomas Uher, ${ }^{30}$ Davide Maimone, ${ }^{31}$ Recai Turkoglu, ${ }^{32}$ Raymond MM Hupperts, ${ }^{33,34}$ Maria Pia Amato, ${ }^{35}$ Franco Granella, ${ }^{36}$ Celia Oreja-Guevara, ${ }^{37}$ Ayse Altintas, ${ }^{38}$ Richard Macdonell, ${ }^{39}$ Tamara Castillo-Trivino, ${ }^{1,2}$ Helmut Butzkueven, ${ }^{40}$ Raed Alroughani, ${ }^{1,2}$ Vilija G Jokubaitis, ${ }^{41}$ MSBase Registry. ${ }^{1}$ Department of Neuroscience, Central Clinical School, Monash University, Melbourne, VIC, Australia; ${ }^{2}$ Multiple Sclerosis and Neuroimmunology Unit, Department of Neurology, Alfred Health, Melbourne, VIC, Australia; ${ }^{3}$ Department of Neurology and Center of Clinical Neuroscience, First Faculty of Medicine, Charles University and General University Hospital, Prague, Czech Republic; ${ }^{4}$ Dokuz Eylul University, Turkey; ${ }^{5}$ Hospital Universitario Virgen Macarena, Spain; ${ }^{6}$ CHUM - Hopital Notre Dame, Canada; ${ }^{7}$ Melbourne MS Centre, Royal Melbourne Hospital, Melbourne, VIC, Australia; ${ }^{8} \mathrm{CORe}$, Department of Medicine, University of Melbourne, Melbourne, VIC, Australia; ${ }^{9}$ Department of Medical and Surgical Sciences and Advanced Technologies; GF Ingrassia, University of Catania - AOU Policlinico-San Marco, University of Catania, Italy; ${ }^{10}$ KTU Medical Faculty Farabi Hospital, Turkey; ${ }^{11}$ Mayis University, Medical Faculty, Turkey; ${ }^{12}$ American University of Beirut, Faculty of Medicine, Nehme and Therese Multiple Sclerosis Center, Beirut, Lebanon; ${ }^{13}$ John Hunter Hospital, New Lambton Heights, NSW, Australia; ${ }^{14}$ Neurology Unit, Azienda Ospedaliero-Universitaria of Modena, Modena, Italy; ${ }^{15}$ Brain and Mind Centre, University of Sydney, Sydney, NSW, Australia; ${ }^{16}$ Univ G.d'Annunzio Chieti-Pescara, Italy; ${ }^{17}$ Department of Neurology, São João Universitary Hospital Center, Porto, Portugal; ${ }^{18}$ Royal Brisbane and Women's Hospital, Herston, QLD, Australia; ${ }^{19}$ St Andrews Place, Spring Hill, QLD, Australia; ${ }^{20}$ Centre de réadaptation déficience physique Chaudière-Appalache, Canada; ${ }^{21}$ Nemocnice Jihlava, Czech Republic; ${ }^{22}$ Neuro Rive-Sud, Canada; ${ }^{23}$ IRCCS Mondino Foundation, Pavia, Italy; ${ }^{24}$ AORN San Giuseppe Moscati Avellino, Italy; ${ }^{25}$ Cliniques Universitaires Saint-Luc, Université Catholique de Louvain, Belgium; ${ }^{26}$ Ospedale Generale Provinciale Macerata, Italy; ${ }^{27}$ Liverpool Hospital, Liverpool, NSW, Australia; ${ }^{28}$ Bakirkoy Education and Research Hospital for Psychiatric and Neurological Diseases, Turkey; ${ }^{29}$ Service of Neurology, Hospital Clinic, Institut d'Investigacions Biomediques August Pi i Sunyer (IDIBAPS), and Institut de Neurociències, Universitat de Barcelona, Barcelona, Spain; ${ }^{30}$ Centro Sclerosi Multipla, UOC Neurologia, ARNAS Garibaldi, Catania, Italy; ${ }^{31}$ Haydarpasa Numune Training and Research Hospital, Turkey; ${ }^{32}$ Maaslandziekenhuis, Netherlands; ${ }^{33}$ Department NEUROFARBA, University of Florence, Italy; ${ }^{34}$ IRCCS Fondazione Don Carlo Gnocchi, Florence, Italy; ${ }^{35}$ University of Parma, Italy; ${ }^{36}$ Department of Neurology, Hospital Clínico San Carlos, Departamento de Medicina, Facultad de Medicina, Universidad Complutense de Madrid (UCM) and IdISSC, Madrid, Spain; ${ }^{37}$ Department of Neurology, Koc University School of Medicine, Turkey; ${ }^{38}$ Department of Neurology, Austin Health, Heidelberg, VIC, Australia; ${ }^{39}$ Department of Neurology, Hospital Universitario Donostia, San Sebastian, Spain; ${ }^{40}$ Amiri Hospital, Kuwait; ${ }^{41}$ MSBase Neuro-immunology Registry, Melbourne, VIC, Australia

10.1136/bmjno-2021-ANZAN.4
Objective To investigate pregnancy-related disease activity in a contemporary multiple sclerosis (MS) cohort.

Methods Data were obtained from the MSBase Registry. Term/ preterm pregnancies conceived from 2011-2019 were included (modern cohort). Annualised relapse rates (ARR) were calculated before, during and after pregnancy. Predictors of intrapartum and early postpartum ( $1^{\text {st }} 3$ months) relapse were determined by clustered logistic and Cox regression analyses, respectively.

Results We included 1640 pregnancies from 1452 women. Disease-modifying therapy (DMT) used in the one-year preconception included natalizumab $(n=219)$, fingolimod $(n=147)$, dimethyl fumarate (DMF; $n=57)$ and low-efficacy therapies $(n=845)$. Preconception ARR by DMT class used before conception were: natalizumab, 0.29 (95\% CI 0.22 0.37); fingolimod, 0.37 (0.28-0.49); DMF, 0.24 (0.13-0.41); low-efficacy, 0.29 (0.25-0.33); and none, 0.24 (0.19-0.31). Among women who used fingolimod or natalizumab, ARR increased during pregnancy. Intrapartum ARR decreased in preconception DMF, low-efficacy or no DMT groups. ARR spiked after delivery across all DMT groups. Natalizumab continuation into pregnancy reduced the odds of relapse during pregnancy (OR 0.76 per month [0.60-0.95], $\mathrm{p}=0.017$ ). DMT re-initiation with natalizumab protected against postpartum relapse (HR 0.11 [0.04-0.32], $\quad \mathrm{p}<0.0001)$. Breastfeeding women were less likely to relapse (HR 0.61 [0.41-0.91], $\mathrm{p}=0.016)$.

Conclusion Women with MS prescribed natalizumab or fingolimod preconception had higher rates of intrapartum and postpartum relapse. In women considered to be at high relapse risk, use of natalizumab before pregnancy and continued up to 32-34 weeks gestation, with early re-initiation after delivery is an effective option to minimise relapse risks. Strategies of DMT use have to be balanced against potential foetal/neonatal complications.

\section{PSORIASIS IN MULTIPLE SCLEROSIS: AN AUSTRALIAN PREVALENCE STUDY}

${ }^{1}$ Varitsara Mangkorntongsakul, ${ }^{2}$ Olivia A Charlton, ${ }^{3}$ Kevin Phan, ${ }^{4}$ Ariadna Fontes, ${ }^{1,4}$ John Parratt, ${ }^{1,4}$ Geoff Herkes, ${ }^{5,6,7}$ Saxon D Smith. ${ }^{1}$ The University of Sydney, Sydney, NSW, Australia; ${ }^{2}$ Department of Dermatology, Royal North Shore Hospital, St Leonards, NSW, Australia; ${ }^{3}$ Department of Dermatology, Liverpool Hospital, Liverpool, NSW, Australia; ${ }^{4}$ Department of Neurology, Royal North Shore Hospital, St Leonards, NSW, Australia; ${ }^{5}$ The Dermatology and Skin Cancer Centre, Gosford, NSW, Australia; ${ }^{6}$ Sydney Adventist Hospital Clinical School, Sydney Medical School, The University of Sydney, Sydney, NSW, Australia; 'Department of Dermatology, Sydney Adventist Hospital, Wahroonga, NSW, Australia

\subsection{6/bmjno-2021-ANZAN.5}

Background Multiple Sclerosis (MS) is an immune-mediated, demyelinating disease of the central nervous system. ${ }^{1}$ Although severe psoriasis and psoriasiform dermatitis have been noted in MS patients, the prevalence of psoriasis in these populations is uncertain and has not been explored in the Australian population.

Objectives A pilot study to estimate the prevalence of psoriasis in MS cohorts in the Australian population.

Methods A survey was conducted on 82 MS patients aged 18 and above who attended MS clinics in 2018.

Results Data was recorded for 82 patients. The mean age was 48 years for the entire cohort and 48.0 years (SD \pm 11.30 ) 
for patients diagnosed with psoriasis. The observed prevalence of psoriasis in MS patients was $12.19 \%$ however, the true prevalence is likely to be much higher as various symptoms of psoriasis were reported by a much larger proportion of MS patients. Of the 72 cases without psoriasis, various skin symptoms that were reported were intermittent irritation (for at least 6 months) or erythematous rash (35.4\%), seasonal skin changes (39.0\%) and thickened scaly skin behind ears and scalps (18.3\%). Moreover, $18.3 \%$ had flaky, peeling or scaly skin while $24.4 \%$ experienced dandruff; $17.1 \%$ reported nail changes, and $13.4 \%$ reported a family history of psoriasis. The study also showed that combined psoriasis and eczema was relatively common at $3.7 \%$.

Conclusions In this pilot study there is a high prevalence of psoriasis in patients with MS suggesting an immunopathological association between the two diseases and indicates that further studies should be done to elucidate common mechanisms, and the nature of this phenotype.

\section{REFERENCE}

1. Montalban X, Tintore M, Swanton J, Barkhof F, Fazekas F, Filippi M, et al. MRI criteria for MS in patients with clinically isolated syndromes. Neurology 2010;74 (5):427-34.

\section{COMPARISON OF MULTIPLE DISEASE MODIFYING THERAPIES IN MULTIPLE SCLEROSIS WITH MARGINAL STRUCTURAL MODELS}

\begin{abstract}
1'brahima Diouf, 1,2Charles B Malpas, 1,2 Sifat Sharmin, 3,4,50lga Skibina, ${ }^{4}$ Katherine Buzzard, ${ }^{6,7}$ Jeannette Lechner-Scott, ${ }^{8}$ Michael Barnett, ${ }^{9}$ Suzanne Hodgkinson, ${ }^{10}$ Mark Slee, ${ }^{11}$ Ernest Butler, ${ }^{12,13}$ Pamela McCombe, ${ }^{4,14}$ Anneke van der Walt, ${ }^{15}$ Helmut Butzkueven, ${ }^{16}$ Steve Vucic, ${ }^{15}$ Richard Macdonell, ${ }^{17}$ Cameron Shaw, 1,2Tomas Kalincik. 'CORe, Department of Medicine, University of Melbourne, Melbourne, VIC, Australia; ${ }^{2}$ MS Centre, Department of Neurology, Royal Melbourne Hospital, Melbourne, VIC, Australia; ${ }^{3}$ Department of Neurology, Box Hill Hospital, Melbourne, VIC, Australia; ${ }^{4}$ Monash University, Melbourne, VIC, Australia; ${ }^{5}$ The Alfred Hospital, Melbourne, Australia; ${ }^{6}$ School of Medicine and Public Health, University Newcastle, Newcastle, NSW, Australia; 'Department of Neurology, John Hunter Hospital, Hunter New England Health, Newcastle, NSW, Australia; ${ }^{8}$ Brain and Mind Centre, Sydney, Australia; ${ }^{6}$ Liverpool Hospital, Sydney, NSW, Australia; ${ }^{9}$ Liverpool Hospital, Sydney, NSW, Australia; ${ }^{10}$ Flinders University, Adelaide, SA, Australia; ${ }^{11}$ Monash Medical Centre, Melbourne, VIC, Australia; ${ }^{12}$ University of Queensland, Brisbane, QLD, Australia; ${ }^{13}$ Royal Brisbane and Women's Hospital, Brisbane, QLD, Australia; ${ }^{14}$ Department of Neurology, The Alfred Hospital, Melbourne, VIC, Australia; ${ }^{15}$ Austin Health, Melbourne, VIC, Australia; ${ }^{16}$ Westmead Hospital, Sydney, NSW, Australia; ${ }^{17}$ Geelong Hospital, Geelong, VIC, Australia
\end{abstract}

\subsection{6/bmjno-2021-ANZAN.6}

Background Because of methodological challenges comparisons of multiple treatments in multiple sclerosis cohorts have been limited to pairwise and triple comparisons.

Objectives Extend marginal structural models (MSM) to allow simultaneous comparisons of multiple MS treatments.

Methods We selected patients from the MSBase registry with Clinically Isolated Syndrome and Relapsing-Remitting MS followed for $\geq 1$ year, with $\geq 3$ visits, $\geq 1$ visit per year and exposed to a MS therapy. MSMs were used to compare cumulative hazards of 6-month confirmed worsening and improvement of disability, and the incidence of relapses between treatments. MSMs were continuously re-adjusted for patient age, sex, pregnancy, date from first symptom, prior relapse history and MRI activity. We used MSMs to compare the Average Treatment Effect (ATE), the effect a treatment would have had if the entire study population had been treated with this treatment vs. another treatment. We also estimated the Average Treatment Effect Among the Treated (ATT): comparison an observed effect of a treatment with a counterfactual (not observed) effect of another treatment in the same study population.

Results Among 23687 patients, we compared ATE of glatiramer acetate (reference), interferon b, natalizumab, fingolimod, dimethyl fumarate, and teriflunomide. In ATE, a reduction of relapse frequency was more prominent on natalizumab, followed by fingolimod $(47 \%$ and $24 \%$ respectively, reference: glatiramer acetate) when compared with the other treatments. The ATT models confirmed these observations.

Conclusions Compared to other DMTs natalizumab and fingolimod were associated with superior reduction in relapse frequency than glatiramer acetate, interferon beta, teriflunomide and dimethyl fumarate.

\section{IMMUNOTHERAPY RESPONSIVE NEUROPATHIC PAIN ASSOCIATED WITH LGI1 AND CASPR2 ANTIBODIES}

\begin{abstract}
${ }^{1,2}$ Sudarshini Ramanathan, ${ }^{2}$ Alexander Davies, ${ }^{2}$ Christopher Uy, ${ }^{2}$ Mandy Tseng, ${ }^{2}$ Sofija Paneva, ${ }^{2}$ Sophia Michael, ${ }^{2}$ James Varley, ${ }^{2}$ Sophie Binks, ${ }^{2}$ Andreas Themistocleous, ${ }^{2}$ Yaacov Anziska, ${ }^{2}$ Ana Candalija, ${ }^{2}$ Anushka Soni, ${ }^{2}$ Monika Hofer, ${ }^{1}$ Fabienne Brilot, ${ }^{1}$ Russell C Dale, ${ }^{2}$ John Dawes, ${ }^{2}$ Simon Rinaldi, ${ }^{2}$ David Bennett, ${ }^{2}$ Sarosh R Irani. ${ }^{1}$ Faculty of Medicine and Health, Sydney Medical School, University of Sydney, Concord, NSW, Australia; ${ }^{2}$ University of Oxford, Oxford, UK
\end{abstract}

\subsection{6/bmjno-2021-ANZAN.7}

Objective We evaluated pain in leucine-rich glioma inactivated1(LGI1) and contactin-associated protein2(CASPR2)-antibody positive $(\mathrm{Ab}+)$ patients, to evaluate clinical associations and pathophysiology of treatable pain syndromes.

Methods 108 LGI-Ab+, 33 CASPR2-Ab+, and 6 LGI1/ $\mathrm{CASPR}-\mathrm{Ab}+$ patients were phenotyped. Pain questionnaires were undertaken to identify neuropathic pain using the Douleur Neuropathique(DN4), patient reported outcome measurement information system(PROMIS), and quality of life(EQ5D). Skin biopsies, and serum binding to cell-based assays, sensory neuronal cocultures, and dorsal root ganglion(DRG) cultures were undertaken.

Results 39/147 patients described pain, including 17/33 CASPR2-Ab+(52\%), 20/108 LGI1-Ab+(19\%), and 2 LGI/ CASPR2-Ab+patients. Questionnaires completed in 23/39 $(59 \%)$ revealed comparable DN4 scores $(\mathrm{p}=0.319)$ with $58 \%$ of LGI1-Ab+ and $67 \%$ of CASPR2-Ab + patients having neuropathic pain. Patients rated $>50 \%$ response in $8 / 30(27 \%)$ analgesia trials, versus 20/40(55\%) immunotherapy trials $(p=0.045)$. PROMIS ratings were similar between LGI1-Ab + and CASPR2-Ab+patients at $\operatorname{nadir}(\mathrm{p}=0.662)$, but showed more improvement following immunotherapy in LGI1-Ab $+(p=0.008)$ than CASPR2-Ab+patients $(p=0.125)$. At follow-up(median 57 months) CASPR2-Ab+patients showed more impairment in mobility $(\mathrm{p}=0.014)$, daily activities $(\mathrm{p}=0.019)$, and anxiety/depression $(\mathrm{p}=0.043)$; and lower overall health $(\mathrm{p}=0.019)$ on the EQ5D compared to LGI1$\mathrm{Ab}+$ patients. Intraepidermal nerve fibre density was reduced in 2 LGI1-Ab+ and 1 CASPR2-Ab+patients. Serum immunoglobulin $\mathrm{G}(\mathrm{IgG})$ from 6/16 CASPR2-Ab+patients bound to sensory neuronal cocultures compared to 0/14 LGI1-Ab + patients $(\mathrm{p}=0.019)$ and $0 / 12$ healthy controls. Serum IgG from 10/16 CASPR2-Ab+patients bound to DRG cultures 